\title{
Probing hepatocyte heterogeneity
}

\author{
Cell Research (2015) 25:1181-1182. doi:10.1038/cr.2015.117; published online 25 September 2015
}

To maintain homeostasis, organs replace cells lost through normal cellular turnover, often through the straightforward replication of existing cells. A recent paper in Nature shows that cells in the liver are not equivalent when it comes to their replicative capacity; rather, a subset of hepatocytes defined by the maintenance of active Wnt signaling bears the brunt of responsibility for maintaining liver mass.

Since at least the 1930s, and possibly well before, the mammalian liver has been recognized for its extraordinary ability to regenerate following injury [1]. Over the course of the $20^{\text {th }}$ century, a paradigm emerged which posited that the liver uses two distinct mechanisms for regeneration depending on the mode of injury. According to this paradigm, the liver regenerates via the replication of existing cells following removal of a part of the liver (i.e., partial hepatectomy) and via the expansion and differentiation of a specialized stem/ progenitor cell pool following exposure to toxins, particularly those toxins that interfere with hepatocyte replication [2]. Recently, however, several studies have called into question whether stem/ progenitor cells contribute substantively to liver regeneration, at least in those injury models previously thought to involve progenitors. Hence, liver regeneration may be driven almost entirely by the replication of existing cells (i.e., hepatocytes), regardless of the mode of injury [3]. Nevertheless, such findings still leave open the possibility that hepatocytes differ in their replicative potential.

To address the issue of possible heterogeneity, Wang and colleagues [4] developed techniques for labeling hepatocytes within different portions of the hepatic lobule to follow their capacity for renewal. The lobule represents the main functional unit of the liver and can be divided into three "zones" (Figure 1). Blood enters the liver through portal tracts located in zone 1, and then percolates between sheets of hepatocytes ("sinusoids") through zones 2 and 3 until exiting the liver through the central veins. This arrangement facilitates the exchange of small molecules between the bloodstream and the hepatocytes, subserving the liver's roles in detoxification, metabolic regulation, and synthesis of plasma proteins.

Zonation within the lobule is known to be under the control of Wnt signaling, with Wnt activity highest in zone 3 hepatocytes near the central vein and lowest in zone 1 hepatocytes near the portal tracts $[5,6]$. Based on this, Wang et al. [4] hypothesized that the zone 3 cells might constitute a hepatocyte subset with a heightened replicative potential. Taking advantage of the fact that zone 3 hepatocytes have higher Wnt activity (and hence higher expression of the Wnt target gene Axin2), the authors used an Axin2-CreER mouse strain [7] to label these cells and follow their fate.

Using this approach, Wang et al. made a number of important observations about the dynamics of cellular turnover in the liver. First, they found that pericentral hepatocytes divide at roughly twice the rate as other hepatocytes, with an estimated doubling time of roughly 14 days, confirming suggestive results from a previous study [8]. Second, they found that the labeled cells made up an increased percentage of liver mass over time, expanding from roughly $5 \%$ of liver area a week following labeling to roughly $30 \%$ of liver area a year later. Third, the authors discovered that central vein endothelial cells produce high levels of the soluble Wnt ligands Wnt2 and Wnt9b. Wnt signaling in the pericentral cells was required for their enhanced proliferation, suggesting that central vein-derived Wnts might define a "niche" for fast-replicating pericentral cells. Finally, Wang et al. showed that in contrast to most hepatocytes, which are polyploid and contain a $4 \mathrm{~N}$ or $8 \mathrm{~N}$ complement of DNA, the majority of Axin2+ hepatocytes are diploid. This latter finding was interpreted as an explanation for the enhanced replicative activity of pericentral hepatocytes; however, other studies have shown that diploid and polyploid hepatocytes have equivalent growth rates in vivo [9].

In light of these findings, it is enticing to think that this population of pericentral hepatocytes might be the long sought-after "liver stem cells". Indeed, other work has shown that in addition to robust self-renewal properties, hepatocytes have the capacity to differentiate into cholangiocytes, a distinct cell type [10]. According to the formal definition of a stem cell - a cell which has the capacity to both self-renew and give rise to another lineage - pericentral hepatocytes would thus appear to fit the bill. Paradoxically, however, pericentral hepatocytes are uniquely incompetent incompetent in terms of their ability to become cholangiocytes [11], suggesting that there is a tradeoff in a cell's ability to replicate vs (trans)-differentiate.

Because lineage tracing studies, par- 


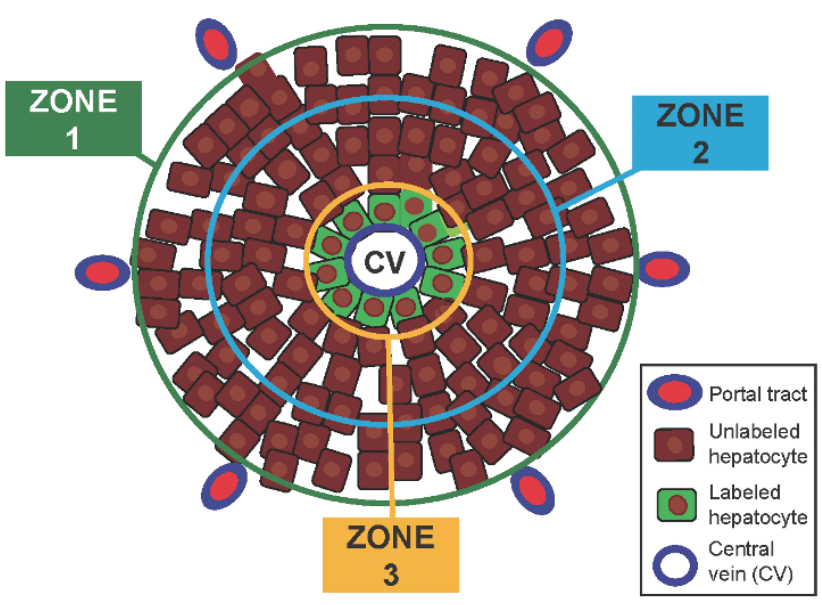

Figure 1 Specialized hepatocytes in the pericentral region of the hepatic lobule. The hepatic lobule is represented as a hexagon, with blood entering through portal tracts at the periphery and converging on a central vein (CV) at the epicenter. As blood makes its way inward, it passes through three "zones" of hepatocytes. As shown by Wang et al., central vein endothelial cells release Wnt ligands, resulting in the activation of Wnt signaling in hepatocytes adjacent to the central vein (zone 3). When labeled with a Wnt-responsive reporter (illustrated in green), these cells exhibited a heightened ability to replicate, with label subsequently appearing in hepatocytes in zone 2 (and even zone 1) of the liver.

ticularly those that rely on Cre recombinase, can be misleading, the results of Wang et al. will need to be confirmed using independent approaches. Nevertheless, the findings raise a number of stimulating questions. How does Wnt signaling govern hepatocyte replication and other features of hepatocyte behavior? Why are pericentral hepatocytes enriched for diploid cells if euploidy does not result in a growth advantage for hepatocytes, and what are the factors that determine replicative capacity? And finally, how do pericentral hepatocytes factor in during liver regeneration, when the liver must sense, and return to, an appropriate size? Regardless of the answers to these questions, this study points to the importance of considering heterogeneity within the pool of differentiated cells — and not just stem cells - when evaluating mechanisms of tissue homeostasis and regeneration.

\section{Ben Z Stanger ${ }^{1}$}

${ }^{1}$ Departments of Medicine and Cell and Developmental Biology, BRB II/III, Rm. 512, 421 Curie Boulevard, Perelman School of Medicine at the University of Pennsylvania, Philadelphia, PA19104

Correspondence: Ben Z Stanger

E-mail: bstanger@exchange.upenn.edu

\section{References}

1 Higgins GM, Anderson RM. Arch Pathol 1931; 12:186-202.

2 Stanger BZ. Annu Rev Physiol 2015; 77:179-200.

3 Grompe M. Cell Stem Cell 2014; 15:257258.

4 Wang B, Zhao L, Fish M, et al. Nature 2015; 524:180-185.

5 Benhamouche S, Decaens T, Godard C, et al. Dev Cell 2006; 10:759-770.

6 Yang J, Mowry LE, Nejak-Bowen KN, et al. Hepatology 2014; 60:964-976.

7 van Amerongen R, Bowman AN, Nusse R. Cell Stem Cell 2012; 11:387-400.

8 Magami $\mathrm{Y}$, Azuma T, Inokuchi $\mathrm{H}$, et al. Liver 2002; 22:419-425.

9 Duncan AW, Taylor MH, Hickey RD, et al. Nature 2010; 467:707-710.

10 Michalopoulos G, Barua L, Bowen W. Hepatology 2005; 41:535-544.

11 Yanger K, Zong Y, Maggs LR, et al. Genes Dev 2013; 27:719-724. 